\title{
Accuracy of Cluster Model Calculations for Quasicrystal Surface
}

\author{
Masanori Sato $^{1, * 1}$, Takanobu Hiroto ${ }^{2}$, Yoshitaka Matsushita ${ }^{2}$ and Kazuki Nozawa ${ }^{1, * 2}$ \\ ${ }^{1}$ Department of Physics and Astronomy, Kagoshima University, Kagoshima 890-0065, Japan \\ ${ }^{2}$ Research Network and Facility Services Division, National Institute for Materials Science, Tsukuba 305-0047, Japan
}

Accuracy of the adsorption energy on a quasicrystal surface calculated with the density functional theory and cluster models were discussed. Two types of cluster models of various sizes were tested to represent a fivefold surface of the Ag-In-Yb quasicrystal. Some cylindrical clusters caused unnaturally rippled potential energy surfaces regardless of the cluster thickness. However, it was confirmed that the ripples are disappeared with a cluster radius of $1.4 \mathrm{~nm}$ or larger. A similar trend was observed even in the hemispherical clusters, and also confirmed in their root mean square errors. It was concluded that both cluster models with a certain size are expected to give relative adsorption energy within an error of $0.15 \mathrm{eV}$. [doi:10.2320/matertrans.MT-MB2020015]

(Received October 9, 2020; Accepted December 21, 2020; Published February 25, 2021)

Keywords: hypermaterials, quasicrystal surface, first-principles calculation, cluster model, adsorption, silver indium ytterbium

\section{Introduction}

The surfaces of quasicrystals (QCs) have been studied extensively since around 1990 when specimens large enough for surface studies begun to be obtained. In the first decade, the atomic structure of clean surfaces of Al-based QCs were studied intensively, and characteristic structures related to the quasiperiodicity such as step height sequence corresponding to a Fibonacci sequence were found. ${ }^{1,2)}$ Furthermore, it was confirmed in many systems that surfaces of QC recover the same quasiperiodicity with its bulk layer after appropriate surface treatment by sputtering and annealing. ${ }^{3-5)}$ The fact that the atomic structure of most of QC surfaces can be considered equivalent to the one of its bulk made us possible to use QC surfaces as a template of crystal growth. In the next decade, several studies reported a quasiperiodic singleelement monolayer formed on Al-based quasicrystals. ${ }^{6-10)}$ Following these studies, three-dimensional single-element quasiperiodic structures composed of multiple $\mathrm{Pb} / \mathrm{Bi}$ layers were succeeded to synthesize on a $\mathrm{Ag}-\mathrm{In}-\mathrm{Yb}$ quasicrystal. ${ }^{11,12)}$ Surprisingly, in these systems, it was confirmed that the deposited $\mathrm{Pb} / \mathrm{Bi}$ atoms occupy the atomic sites of the substrate, meaning the deposited layers were growing with rebuilding the substrate quasicrystal truncated at the surface.

For this kind of study, generally, calculations based on the density functional theory (DFT) play an important role in elucidating the adsorption structure. However, because the conventional DFT calculations cannot handle the aperiodic atomic arrangement of QCs, we need to approximate the QC surfaces in some way. One is using an approximant crystal, a periodic crystal with a close composition and similar local atomic arrangement with the corresponding quasicrystalline phase. In this method, we can carry out DFT calculations without any special treatment because the approximant crystals are periodic. However, the structure of the approximant crystals is, strictly speaking, different from that of QCs. The other approach is the cluster model, where an atomic cluster extracted from the structural data of QCs is used. In this method, the local quasiperiodic atomic

\footnotetext{
${ }^{* 1}$ Graduate Student, Kagoshima University

${ }^{* 2}$ Corresponding author, E-mail: kazuki.nozawa@gmail.com
}

arrangement of QC surfaces can be handled in conventional DFT calculations because the cluster is encapsulated in a periodically arranged large supercell. The cluster model is used even for studies of periodic crystal surfaces, and it is known that the embedding technique reduces the size of the cluster. ${ }^{13)}$ However, it is difficult for QC surfaces to obtain a reliable external potential representing the effect of the environmental atoms. Since there have been only a few reports on the cluster model for the QC surfaces so far, ${ }^{14-16)} \mathrm{a}$ detailed study on the convergence of calculation concerning the cluster size is required. In this paper, we present adsorption energies of a Bi adatom calculated by DFT calculations at various positions on the fivefold surface of icosahedral Ag-In-Yb QC and discuss the convergence behavior of the adsorption energy with respect to the cluster size. We also mention the adequate size of the cluster for computing the potential energy surfaces (PESs), which is used to determine the adsorption pathway.

\section{Models and Calculation}

The icosahedral Ag-In- $\mathrm{Yb}$ QC is isostructural to $\mathrm{Cd}_{5.7} \mathrm{Yb}$ binary QC. The structure can be described as a quasiperiodic arrangement of a structural building block called Tsai-cluster composed of successive atomic shells made of several polyhedra. ${ }^{17)}$ Except for few atoms located in the interstitial region between Tsai-clusters, $\mathrm{Yb}$ atoms occupy an icosahedral shell, and Cd atoms occupy the other shells. Although the atomic positions of $\mathrm{Cd}_{5.7} \mathrm{Yb}$ QC has been solved unambiguously, the exact occupancy of $\mathrm{Ag}$ and $\mathrm{In}$ in $\mathrm{Ag}-$ $\mathrm{In}-\mathrm{Yb} \mathrm{QC}$ is unknown because $\mathrm{Cd}$ sites in $\mathrm{Cd}-\mathrm{Yb} \mathrm{QC}$ are occupied by Ag or In atoms in the ternary Ag-In-Yb QC. To avoid the fractionally occupied sites, we assumed a chemical ordering for $\mathrm{Ag}$ and $\mathrm{In}$ according to the occupation probability deduced from X-ray diffraction experiment for the cubic approximant. ${ }^{18)}$

The DFT calculations were carried out with Vienna Abinitio Simulation Package. ${ }^{19,20)}$ The projector-augmented wave potentials were used to describe the effective interaction between the electron and ionic core. ${ }^{21,22)}$ The generalized gradient approximation was adopted for the exchangecorrelation term. ${ }^{23}$ ) The $4 \mathrm{f}$-electrons of the $\mathrm{Yb}$ atom were 
(a)

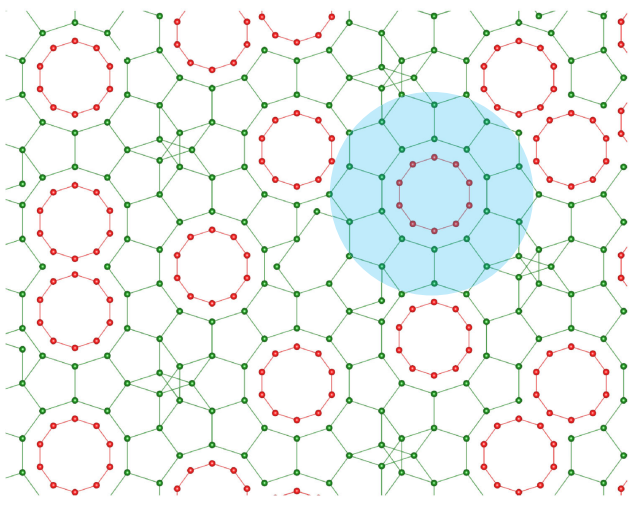

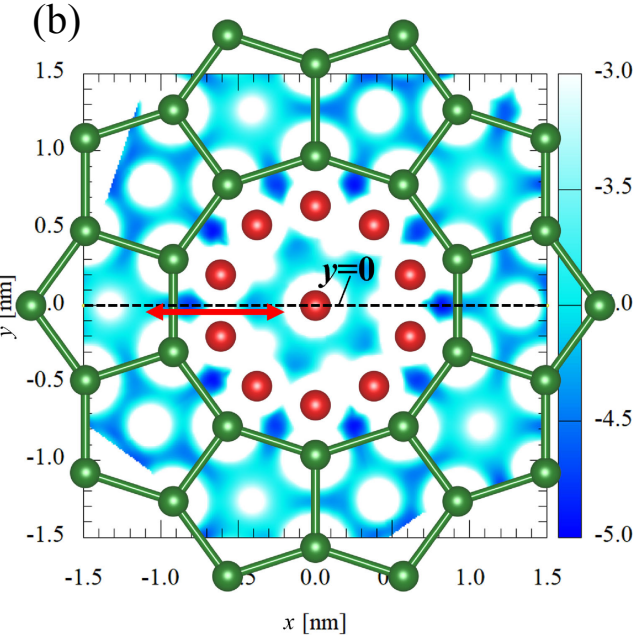

Fig. 1 (a) Surface top layer of the icosahedral Ag-In-Yb quasicrystal. The red and green spheres denote In and Yb atoms. (b) The shaded area in (a). Red arrow indicates a region used for calculations.

kept frozen in the core, and the spin-polarization effect was neglected. Although the vdW-DF functional was applied to check the effect of the long-range dispersion interaction, no significant improvement was obtained. Therefore, in the following, we describe the results without using this functional. $^{24-27)}$

An intensive study of the fivefold surface of the i-AgIn-Yb QC using the scanning tunneling microscope (STM) revealed that the surface is formed at bulk planes intersecting the center of the Tsai-clusters. ${ }^{5)}$ The experiment also reported that the surface plane is atomically flat and Yb-rich. Figure 1(a) shows the top view of a surface that satisfies these experimental observations. Red and green spheres in Fig. 1(a) denote $\mathrm{Ag} / \mathrm{In}$ and $\mathrm{Yb}$ sites, respectively. Assuming the chemical ordering mentioned above, Ag atoms seldom appear in the surface top layer. Actually, all the red spheres shown in Fig. 1(a) are In atoms. In this paper, we focus on a typical structure shaded blue in Fig. 1(a), where the formation of first- and second-layers of $\mathrm{Pb} / \mathrm{Ag}-\mathrm{In}-\mathrm{Yb}$ and $\mathrm{Bi} / \mathrm{Ag}-\mathrm{In}-\mathrm{Yb}$ systems were observed.

In this paper, we approximate the QC surface using two types of cluster models. In both models, precise adsorption energy cannot be calculated at the edge of the cluster, where surface atoms are in unrealistic electronic states. Large clusters are used for periodic surfaces to avoid using the cluster edge for calculating the adsorption energy. However, it may require a colossal cluster to explore a vast surface area. Alternatively, we may define the clusters so that the adsorption sites are always at the cluster center. This method requires a dedicated cluster for each adsorption site but enables us to reduce the cluster radius. The adsorption energy $E_{\text {ads }}$ is represented as

$$
\begin{aligned}
E_{\text {ads }}(x, y, z)= & E_{\text {surface+adsorbate }}(x, y, z) \\
& -\left[E_{\text {surface }}(x, y, 0)+E_{\text {adsorbate }}\right],
\end{aligned}
$$

where $(x, y)$ are the position of the adsorbate in the surface parallel directions, and $z$ is the distance between the adsorbate and surface top layer. Thus, $\boldsymbol{r}^{\prime}=(x, y, 0)$ specifies a position on the surface top layer just below the adsorbate at $\boldsymbol{r}=(x, y, z)$, and $E_{\text {surface+adsorbate }}(\boldsymbol{r}), E_{\text {surface }}\left(\boldsymbol{r}^{\prime}\right), E_{\text {adsorbate }}$ are (a)
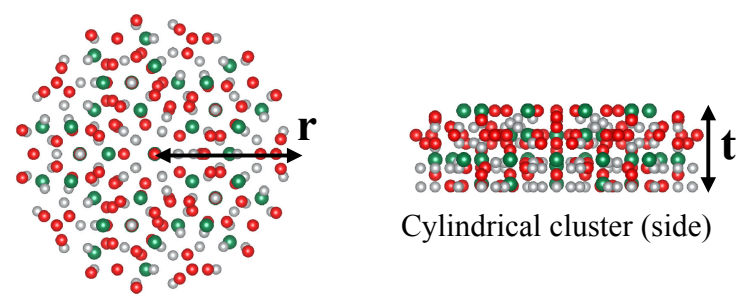

Cylindrical cluster (side)

Cylindrical cluster (top)

(b)
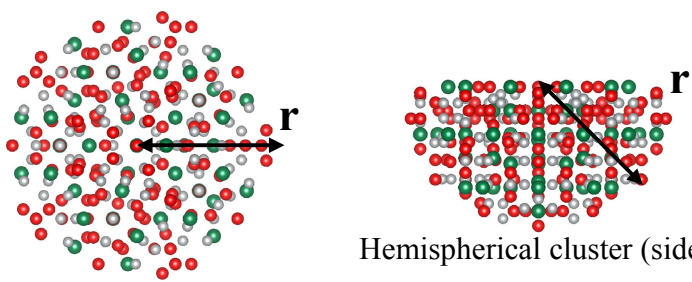

Hemispherical cluster (side)

Hemispherical cluster (top)

Fig. 2 The cylindrical (a) and hemispherical (b) cluster model. The red, green, and silver spheres represent $\mathrm{In}, \mathrm{Yb}$, and $\mathrm{Ag}$ atoms, respectively.

the total energies of the adsorbed system, clean surface and isolated $\mathrm{Bi}$ adatom, respectively. Note that this method requires $E_{\text {surface }}\left(\boldsymbol{r}^{\prime}\right)$ for each adsorption site.

We tested cylindrical and hemispherical clusters displayed in Fig. 2(a) and (b). The cylindrical cluster is described by the radius $(r)$ and thickness $(t)$, whereas the single parameter $r$ specifies the hemispherical cluster. For simplicity, we denote cylindrical clusters as $\mathrm{rRtT}$, where $\mathrm{R}$ and $\mathrm{T}$ are the radius and thickness of the cluster in $10^{-1} \mathrm{~nm}$, respectively. Similarly, a hemispherical cluster with radius $\mathrm{R} \times 10^{-1} \mathrm{~nm}$ is denoted as hrR. For example, r15t8 refers to the cylindrical cluster with a radius of $1.5 \mathrm{~nm}$ and a thickness of $0.8 \mathrm{~nm}$, and hrl2 refers to the hemispherical cluster with a radius of $1.2 \mathrm{~nm}$. The distance between the $\mathrm{Bi}$ adatom and the substrate is represented by a parameter $z$. Since it has been experimentally and theoretically confirmed that the first layer of this system is formed about $0.1 \mathrm{~nm}$ above the topmost layer 
of the surface, we tested $z=0.10,0.14,0.18$, and $0.22 \mathrm{~nm}$. The adsorption energies were calculated on a segment $y=0,-1.1 \leq x \leq-0.2 \mathrm{~nm}$ with an interval of $0.02 \mathrm{~nm}$, containing forty-seven sampling points on this segment. The region considered is indicated by a red arrow in Fig. 1(b).

\section{Results and Discussions}

We show some of the PESs for the Bi adatom 0.10 and $0.18 \mathrm{~nm}$ away from the surface in Figs. 3. The PES was interpolated using the cubic spline method, which approximates each interval between the PES data points by a polynomial of degree three. Each coefficient in a polynomial function is determined so that both the first and second derivatives are continuous at each data point. Unnatural ripples of the PESs correlate with the insufficient radius of the cluster. As shown in Fig. 3(a), even the thinnest cluster $(\mathrm{t}=0.4 \mathrm{~nm})$ shows a smooth curve if it has a large radius $(\mathrm{r}=2.0 \mathrm{~nm})$, whereas even the thickest cluster $(\mathrm{t}=1.8 \mathrm{~nm})$ shows rippled PES in the case of the radius of which is small $(\mathrm{r}=1.0 \mathrm{~nm})$ as displayed in Fig. 3(b). Figure 3(c) and (d) show a non-uniform dependence of the PES on the cluster radius and thickness. The adsorption energies are well converged at most positions in Fig. 3(c), but a cluster radius dependence is found around $x=-10.5 \mathrm{~nm}$. Similarly, a cluster thickness dependence is found around $x=-10.0 \mathrm{~nm}$ and $x=-4.5 \mathrm{~nm}$ in Fig. 3(d). We shall return to these cluster size dependences later. As one of them is found in Fig. 3(c), some adsorption sites have very close adsorption energies. Thus, the relative adsorption energy should be converged within $0.1-0.2 \mathrm{eV}$ to predict the correct adsorption structure.

Root mean square errors (RMSEs) of cylindrical clusters between the largest cluster ( $\mathrm{t} 18 \mathrm{r} 20)$, which were evaluated at the forty-seven points, are presented in Figs. 4(a)-(d) for adsorption heights $z=0.10-0.22 \mathrm{~nm}$. In these figures, a sparse distribution in the vertical direction means a large radius dependence, and a large slope of a line means a large thickness dependence. The RMSE of the t18r20 cluster is zero because the RMSE was evaluated with respect to this cluster. Clusters with larger radius tend to show smaller RMSE values. However, the RMSE does not decrease monotonically but an oscillatory decrease after a sudden drop between 0.4 and $0.6 \mathrm{~nm}$ thickness. There seem to be two main causes of the error. One of them is an insufficient cluster radius, as mentioned above. The PES ripples caused by this error are improved with a radius of $1.2 \mathrm{~nm}$ and almost disappeared with around $\mathrm{r}=1.4 \mathrm{~nm}$. This trend can also be found in Figs. 4, where the RMSEs of $\mathrm{r}=1.2 \mathrm{~nm}$ or larger form a bunch of lines, meaning lesser radius dependence. Another cause of the error is supposed to be from a manybody effect. A larger cluster should give larger adsorption energy in the pairwise description because interactions between the adatom and the extra atoms compared with a smaller cluster are always attractive. However, we can find some examples that a smaller cluster gives larger adsorption energy. Figure 3(c) shows the t14r16 cluster gives larger adsorption energy than the $114 \mathrm{r} 20$ cluster around $x=$ $-10.5 \mathrm{~nm}$. Similarly, the $\mathrm{t} 16 \mathrm{r} 20$ cluster gives larger adsorption energy than the $118 \mathrm{r} 20$ cluster in a range between
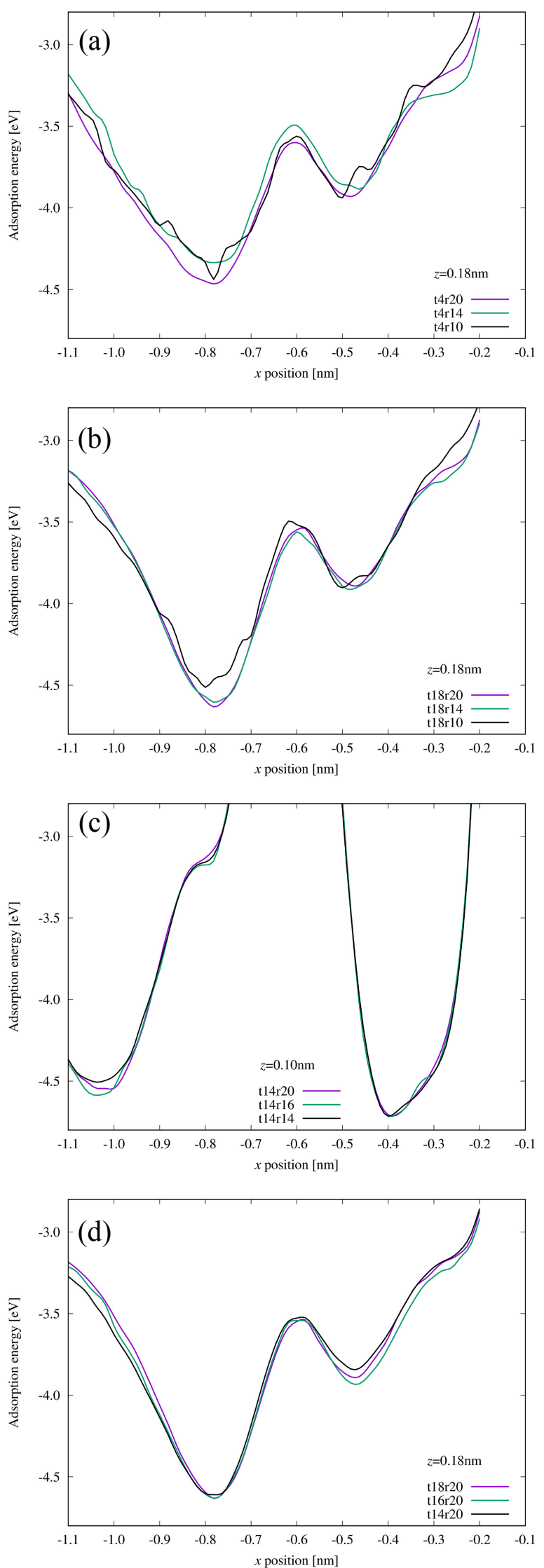

Fig. 3 (a)-(d) Potential energy surfaces calculated with various sizes of clusters.

$x=-5$ to $x=-2 \mathrm{~nm}$, as displayed in Fig. 3(d). This effect seems to make the RMSE convergence slow, and it also affects the value of the adsorption energy around local 

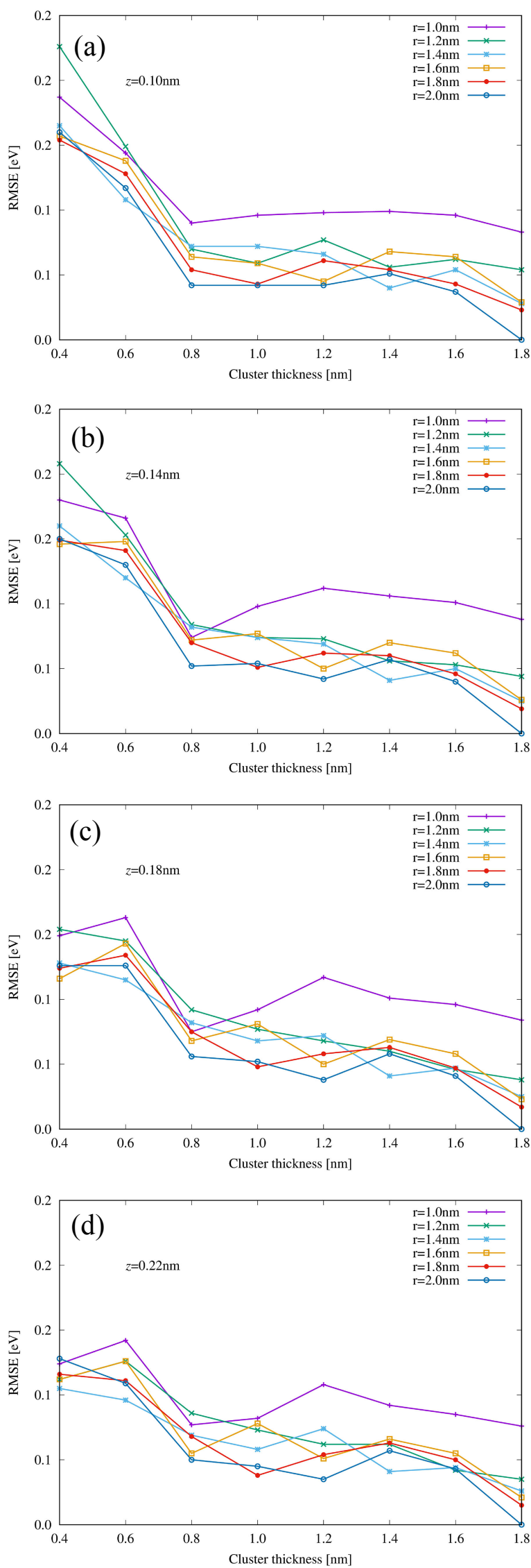

Fig. 4 The root-mean-square error of the adsorption energy of the cylindrical cluster model evaluated with various adsorption heights.
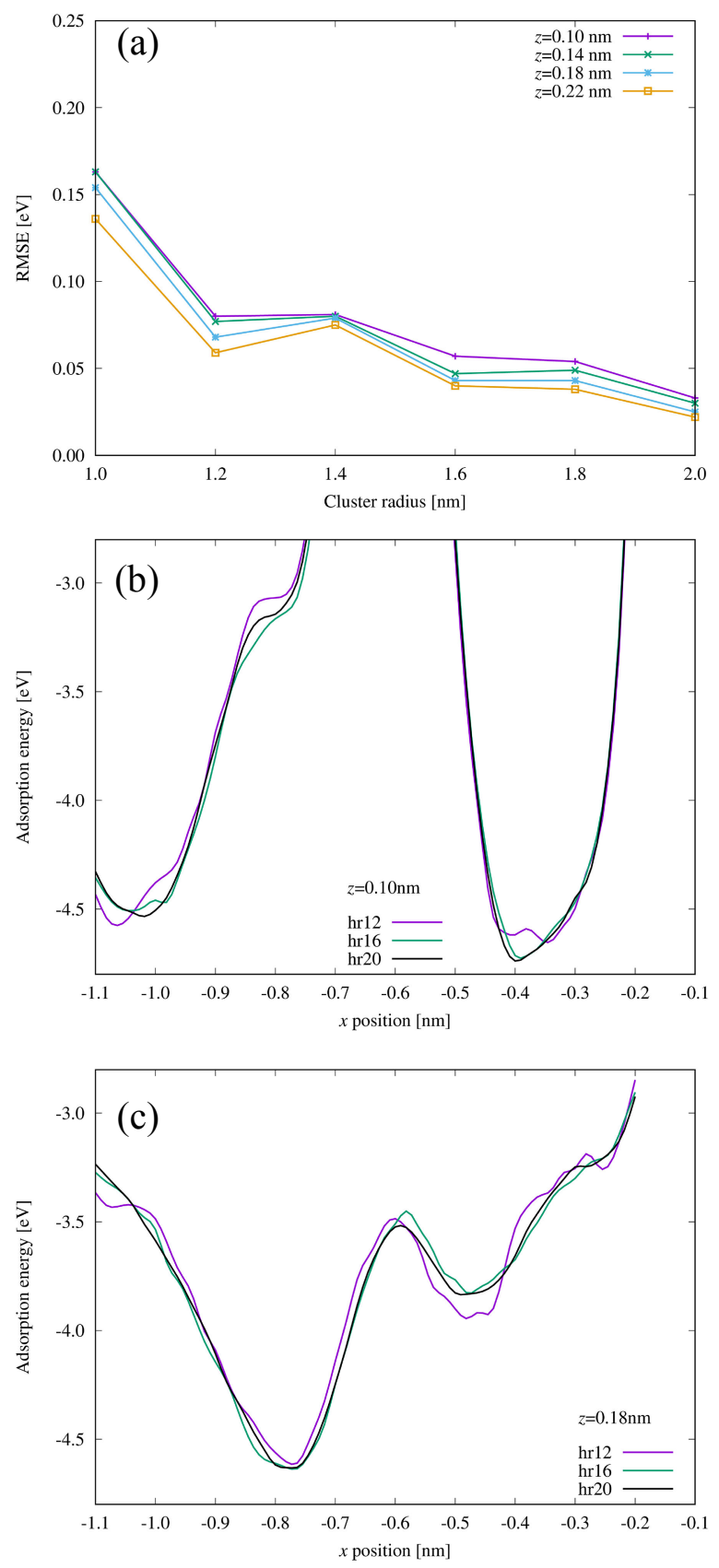

Fig. 5 (a) The root-mean-square error and (b), (c) potential energy surfaces of the hemispherical cluster model.

minima. This might be relevant to the aperiodic atomic arrangement of the QC surface. It is required similar calculations using the same model cluster for periodic crystals to confirm the effect of the aperiodicity.

Because the RMSE of $0.4 \mathrm{~nm}$ thickness clusters decreases with increasing $z$, the abrupt change from $\mathrm{t}=0.4 \mathrm{~nm}$ to $\mathrm{t}=0.6 \mathrm{~nm}$ found in the result of small $z$ (Figs. 3(a) and (b)) becomes smaller with larger $z$. From this, it can be supposed that the effect of the insufficient thickness is reduced in the case of the adsorbate is away from the surface. However, it seems not to mean that the cluster thickness is less important at larger $z$. The slope of the lines between $0.6 \mathrm{~nm}$ to $1.8 \mathrm{~nm}$ looks larger at larger $z$. The thickness dependence is found even between the large clusters like t16r18 and t18r18. On the other hand, only small differences are found between 18 
(a)

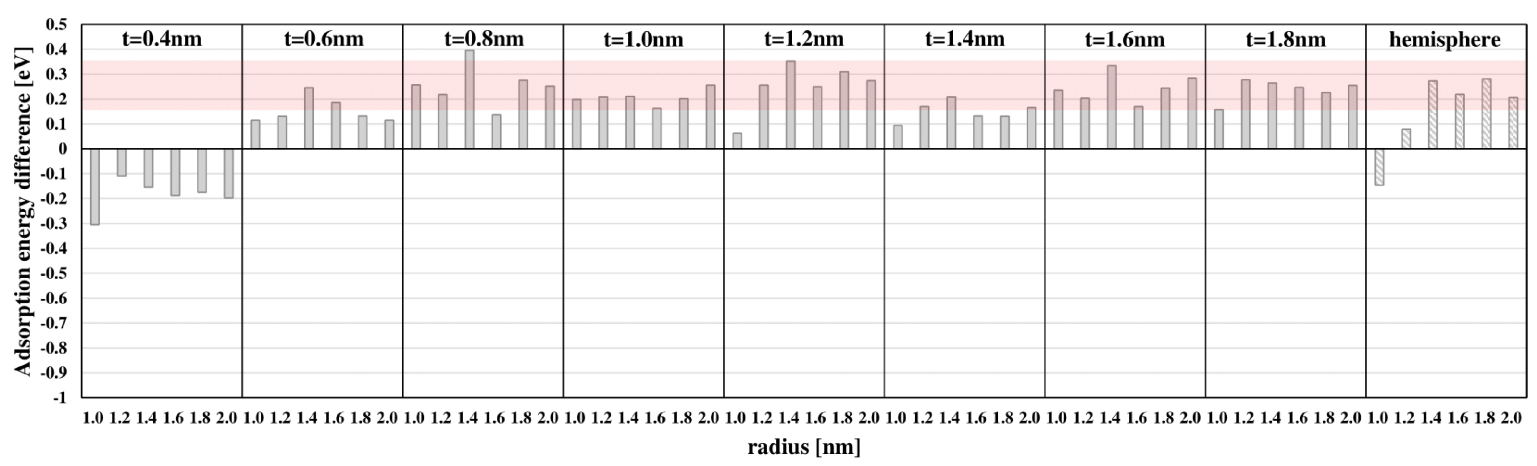

(b)

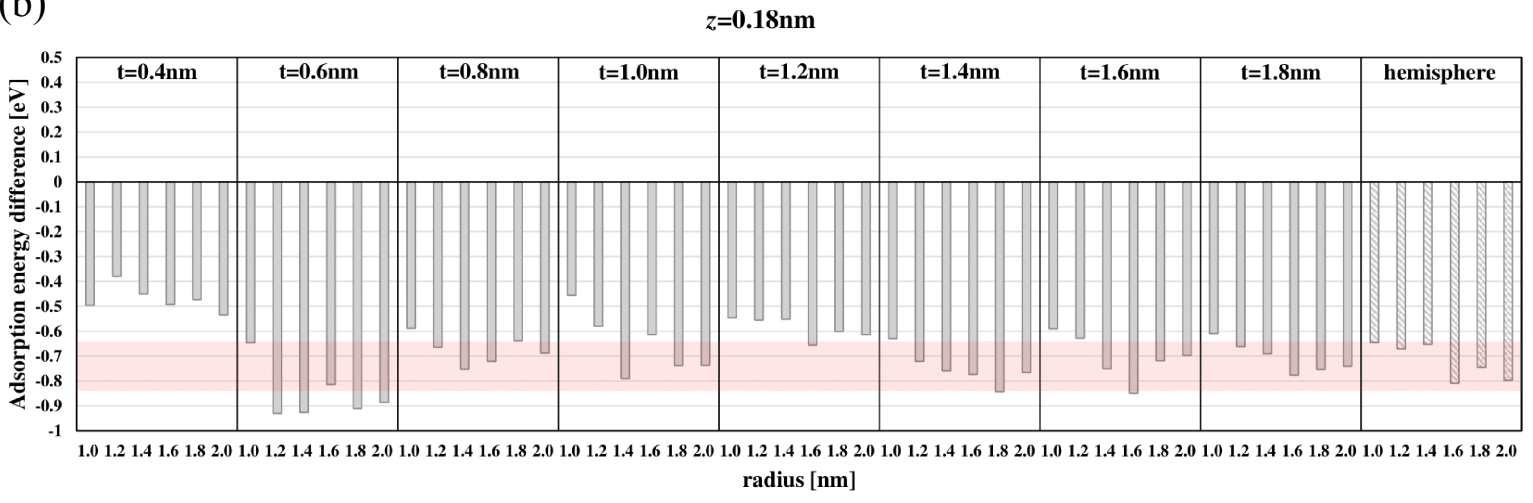

Fig. 6 Relative adsorption energies at (a) $z=0.10$ and (b) $0.18 \mathrm{~nm}$.

and r20 clusters. The RMSE seems to be sufficiently converged with respect to the cluster radius.

The RMSEs of hemispherical clusters are shown in Fig. 5 as a function of the cluster radius. The adsorption energies of the hr22 cluster were used as a reference to evaluate the RMSE. In the hemispherical cluster model, the distance between the $\mathrm{Bi}$ adatom and the truncated edge of the cluster is $\sqrt{r^{2}+z^{2}}$ for the nearest edge and $r+z$ for the farthest edge, where $r$ and $z$ are the radius of the hemisphere and the adsorption height of the $\mathrm{Bi}$ adatom, respectively. Because $r$ is about 5-20 times larger than $z$, the distance between the $\mathrm{Bi}$ adatom and any position on the cluster's hemispherical surface is approximately the same.

The RMSE decreases with increasing the cluster radius. The RMSE drops abruptly between 1.0 and $1.2 \mathrm{~nm}$, and slightly smaller RMSE values are obtained at larger $z$. The hr12 and hr14 clusters and hr16 and hr18 clusters take relatively close RMSE values, but the RMSEs decrease continuously up to $r=2.0 \mathrm{~nm}$. We show the PES of the hemispherical clusters in Figs. 5(b) and (c) for $z=0.10$ and $0.18 \mathrm{~nm}$, respectively. Rippled surfaces similar to that we have seen in the PES of the cylindrical clusters are found again, and the ripples in the PES were suppressed with increasing the radius of the hemisphere.

We have so far discussed the averaged property of the adsorption energy. However, another important quantity to determine the adsorption structure or, more generally the adsorption pathway, is the adsorption energy difference $(\Delta E)$ between different positions. As one example of $\Delta E$, energy differences between the two energy minima at $z=0.10$ and
$0.18 \mathrm{~nm}$ shown in Figs. 3(c) and (d) were evaluated and summarized in Figs. 6(a) and (b), respectively. The adsorption energy difference $\Delta E$ was defined as negative when the left-side minimum is more stable than the right-side minimum. Because the adsorption energies were calculated on a grid, the interval of which is $0.2 \mathrm{~nm}$, we used interpolated values using the cubic spline method for evaluating the relative adsorption energy.

At $z=0.10 \mathrm{~nm}, \Delta E \mathrm{~s}$ of cylindrical clusters with $0.4 \mathrm{~nm}$ thickness and the hemispherical cluster with $1.0 \mathrm{~nm}$ radius gave an opposite sign to that of the other clusters. However, as expected from the RMSE discussed above, it was improved at $z=0.18 \mathrm{~nm}$. The shaded areas in Figs. 6 indicate ranges of $\pm 0.1 \mathrm{eV}$ centered at the value of the t18r20 cluster for a guide to the eye. Most of the clusters larger than t6r16 or hr14 give $\Delta \mathrm{E}$ differing less than $0.15 \mathrm{eV}$ with the 118 r20 cluster. Larger clusters gave better results, but such calculations are unrealistic for constructing the PESs to seek the adsorption pathways. From this viewpoint, small clusters like the t6r16 or hr14 clusters are supposed to be enough. Cross-checking with a calculation using a different model is needed to discuss the stability of adsorption sites, the energy difference of which is less than $0.15 \mathrm{eV}$. The number of atoms included in a cluster is displayed in Fig. 7 as a function of the cluster radius. The number of atoms in a cluster also depends on the cluster center's position. The number of atoms displayed in Fig. 7 is of the cluster centered at the origin of Fig. 1(b). Comparing the t6r16 and hr14 clusters, the number of atoms the t6r16 cluster contains is slightly less than that of the hr14 cluster. In this case, the 


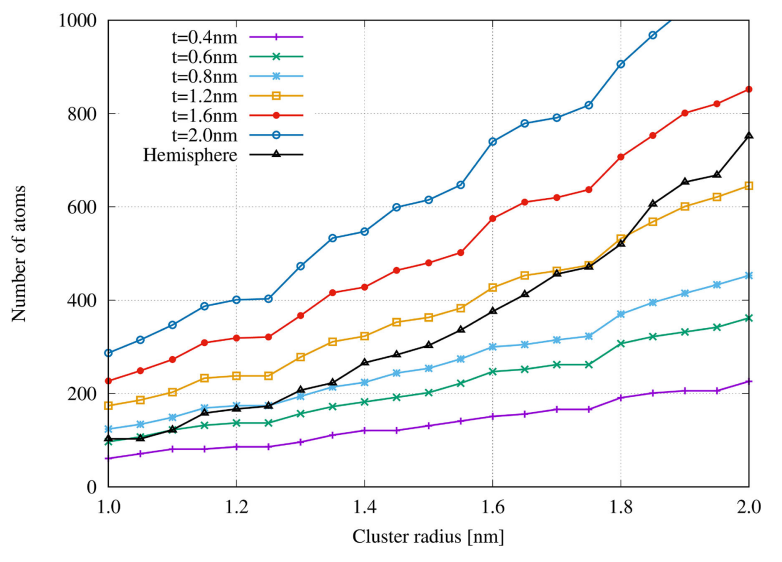

Fig. 7 A relation between the size of the cluster and the number of atoms included in the cluster.

t6r16 cluster is economical in terms of the calculation cost because of the small number of atoms the cluster contains and the small size of the supercell.

\section{Summary}

Accuracy of the adsorption energy on a QC surface calculated with the DFT method and cluster models were discussed. Two types of cluster models of various sizes were tested to represent a fivefold surface of the Ag-In-Yb QC. Some cylindrical clusters caused unnaturally rippled PESs regardless of the cluster thickness, but it was confirmed that the ripples are disappeared with a cluster radius of $1.4 \mathrm{~nm}$ or larger. A similar trend was observed even in the hemispherical clusters and also confirmed in their RMSEs. It was concluded that both cluster models with a certain size are expected to give relative adsorption energy within an error of $0.15 \mathrm{eV}$.

\section{Acknowledgments}

K.N. thanks Dr. H.R. Sharma, Prof. R. McGrath, Prof. K. Makoshi, and Prof. Y. Ishii for fruitful discussions. This work was supported by JSPS KAKENHI Grant Numbers JP17K05059, 18KK0390, 19H05817, and 19H05820. Numerical calculations were carried out using supercomputers at the National Institute for Materials Science, Japan, the Institute for solid state physics at the University of Tokyo, and Yukawa Institute at Kyoto University.

\section{REFERENCES}

1) A.R. Kortan, R.S. Becker, F.A. Thiel and H.S. Chen: Phys. Rev. Lett. 64 (1990) 200-203.

2) T.M. Schaub, D.E. Burgler and H.J. Guntherodt: Phys. Rev. Lett. 73 (1994) 1255-1258.

3) J. Ledieu, R. McGrath, R.D. Diehl, T.A. Lograsso, D.W. Delaney, Z. Papadopolos and G. Kasner: Surf. Sci. 492 (2001) L729-L734.

4) H.R. Sharma, V. Fournáee, M. Shimoda, A.R. Ross, T.A. Lograsso, A.P. Tsai and A. Yamamoto: Phys. Rev. Lett. 93 (2004) 165502.

5) H.R. Sharma, M. Shimoda, K. Sagisaka, H. Takakura, J.A. Smerdon, P.J. Nugent, R. McGrath, D. Fujita, S. Ohhashi and A.P. Tsai: Phys. Rev. B 80 (2009) 121401.

6) K.J. Franke, H.R. Sharma, W. Theis, P. Gille, Ph. Ebert and K.H. Rieder: Phys. Rev. Lett. 89 (2002) 156104.

7) J. Ledieu, L. Leung, L.H. Wearing, R. McGrath, T.A. Lograsso, D. Wu and V. Fournáee: Phys. Rev. B 77 (2008) 073409.

8) Th. Deniozou, J. Ledieu, V. Fournáee, D.M. Wu, T.A. Lograsso, H.I. Li and R.D. Diehl: Phys. Rev. B 79 (2009) 245405.

9) J.A. Smerdon, L. Leung, J.K. Parle, C.J. Jenks, R. McGrath, V. Fournáee and J. Ledieu: Surf. Sci. 602 (2008) 2496-2501.

10) H.R. Sharma, M. Shimoda, A.R. Ross, T.A. Lograsso and A.P. Tsai: Phys. Rev. B 72 (2005) 045428.

11) H.R. Sharma, K. Nozawa, J.A. Smerdon, P.J. Nugent, I. McLeod, V.R. Dhanak, M. Shimoda, Y. Ishii, A.P. Tsai and R. McGrath: Nat. Commun. 4 (2013) 2715.

12) S.S. Hars, H.R. Sharma, J.A. Smerdon, S. Coates, K. Nozawa, A.P. Tsai and R. McGrath: Surf. Sci. 678 (2018) 222-227.

13) K. Yu, F. Libisch and E.A. Carter: J. Chem. Phys. 143 (2015) 102806, and references therein.

14) K. Nozawa and Y. Ishii: Philos. Mag. 91 (2011) 2913-2919.

15) K. Nozawa and Y. Ishii: J. Phys. Conf. Ser. 809 (2017) 012018.

16) K. Nozawa: Proc. ISCEAS, (2018) pp. 197-206.

17) H. Takakura, C.P. Gómez, A. Yamamoto, M. de Boissieu and A.P. Tsai: Nat. Mater. 6 (2007) 58-63.

18) H.R. Sharma, G. Simutis, V.R. Dhanak, P.J. Nugent, C. Cui, M. Shimoda, R. McGrath, A.P. Tsai and Y. Ishii: Phys. Rev. B 81 (2010) 104205.

19) G. Kresse and J. Hafner: Phys. Rev. B 47 (1993) 558-561.

20) G. Kresse and J. Furthmuller: Phys. Rev. B 54 (1996) 11169-11186.

21) P.E. Blöchl: Phys. Rev. B 50 (1994) 17953-17979.

22) G. Kresse and D. Joubert: Phys. Rev. B 59 (1999) 1758-1775.

23) J.P. Perdew, K. Burke and M. Ernzerhof: Phys. Rev. Lett. 77 (1996) 3865-3868.

24) M. Dion, H. Rydberg, E. Schröder, D.C. Langreth and B.I. Lundqvist: Phys. Rev. Lett. 92 (2004) 246401

25) G. Román-Pérez and J.M. Soler: Phys. Rev. Lett. 103 (2009) 096102.

26) J. Klimeš, D.R. Bowler and A. Michaelides: Phys. Rev. B 83 (2011) 195131.

27) I. Hamada: Phys. Rev. B 89 (2014) 121103. 